\title{
Neutrino-Nuclear Coherent Scattering and the Effective Neutrino Charge Radius
}

\author{
Joannis Papavassiliou* \\ Departamento de Física Teòrica and IFIC Centro Mixto, Universidad de Valencia-CSIC, \\ E-46100, Burjassot, Valencia, Spain \\ E-mail: Joannis.Papavassiliouduv.es

\section{Jose Bernabéu} \\ Departamento de Física Teòrica and IFIC Centro Mixto, Universidad de Valencia-CSIC, \\ E-46100, Burjassot, Valencia, Spain \\ E-mail: Jose.Bernabeu@uv.es

\section{Massimo Passera} \\ Dipartimento di Fisica "G. Galilei”, Università di Padova and INFN, Sezione di Padova, \\ I-35131, Padova, Italy \\ E-mail: massimo.passera@pd.infn.it
}

\begin{abstract}
We propose to extract the value of the effective neutrino charge radius from the coherent scattering of a neutrino against a heavy nucleus. In such an experiment the relevant quantity to measure is the kinetic energy distribution of the recoiling nucleus, which, in turn, may be directly related to the shift in the value of the effective weak mixing angle produced by the neutrino charge radius. This type of experiment has been proposed in order to observe the coherent elastic neutrino-nuclear scattering for the first time. If interpreted in the way suggested in this work, such an experiment would constitute the first terrestrial attempt to measure this intrinsic electromagnetic property of the neutrino.
\end{abstract}

International Europhysics Conference on High Energy Physics

July 21st - 27th 2005

Lisboa, Portugal

${ }^{*}$ Speaker. 
It is well-established by now that the difficulties associated with the definition [1] of the neutrino charge radius (NCR) have been conclusively settled in a series of papers [2, 3, 4], by resorting to the well-defined electroweak gauge-invariant separation of physical amplitudes into effective self-energy, vertex and box sub-amplitudes, implemented by the pinch technique formalism [5]. Thus, within the Standard Model, at one-loop order, the NCR, to be denoted by $\left\langle r_{v_{i}}^{2}\right\rangle$, is given by

$$
\left\langle r_{v_{i}}^{2}\right\rangle=\frac{G_{F}}{4 \sqrt{2} \pi^{2}}\left[3-2 \log \left(\frac{m_{i}^{2}}{M_{W}^{2}}\right)\right]
$$

where $i=e, \mu, \tau$, the $m_{i}$ denotes the mass of the charged iso-doublet partner of the neutrino under consideration, and $G_{F}$ is the Fermi constant. In addition, as has been demonstrated in [3], the NCR so defined can be expressed in terms of a judicious combination of physical cross-sections, a fact which promotes it into a genuine physical observable. This possibility has revived the interest in this quantity [6], and makes the issue of its actual experimental measurement all the more interesting. In this talk we will argue that upcoming experiments involving coherent neutrino-nuclear scattering $[7,8]$ may provide the first opportunity for measuring the NCR (or, at least, for placing bounds on its value).

The notion of coherent nuclear scattering is well-known from electron scattering. In the neutrino case it was developed in connection with the discovery of weak neutral currents, with a component proportional to the number operator [9]. When a projectile (e.g. a neutrino) scatters elastically from a composite system (e.g. a nucleus), the amplitude $F\left(\mathbf{p}^{\prime}, \mathbf{p}\right)$ for scattering from an incoming momentum $\mathbf{p}$ to an outgoing momentum $\mathbf{p}^{\prime}$ is given as the sum of the contributions from each constituent,

$$
F\left(\mathbf{p}^{\prime}, \mathbf{p}\right)=\sum_{j=1}^{A} f_{j}\left(\mathbf{p}^{\prime}, \mathbf{p}\right) e^{i \mathbf{q} \cdot \mathbf{x}_{j}},
$$

where $\mathbf{q}=\mathbf{p}^{\prime}-\mathbf{p}$ is the momentum transfer and the individual amplitudes $f_{j}\left(\mathbf{p}^{\prime}, \mathbf{p}\right)$ are added with a relative phase-factor, determined by the corresponding wave function. The differential crosssection is then

$$
\frac{d \sigma}{d \Omega}=\left|F\left(\mathbf{p}^{\prime}, \mathbf{p}\right)\right|^{2}=\sum_{j=1}^{A}\left|f_{j}\left(\mathbf{p}^{\prime}, \mathbf{p}\right)\right|^{2}+\sum_{j, i}^{i \neq j} f_{i}\left(\mathbf{p}^{\prime}, \mathbf{p}\right) f_{j}^{\dagger}\left(\mathbf{p}^{\prime}, \mathbf{p}\right) e^{i \mathbf{q} \cdot\left(\mathbf{x}_{j}-\mathbf{x}_{i}\right)} .
$$

In principle, due to the presence of the phase factors, major cancellations may take place among the $A(A-1)$ terms in the second (non-diagonal) sum. This happens for $q R \gg 1$, where $R$ is the size of the composite system, and the scattering would be incoherent. On the contrary, under the condition that $q R \ll 1$, then all phase factors may be approximated by unity, and the terms in (3) add coherently. If there were only one type of constituent, i.e. $f_{j}\left(\mathbf{p}^{\prime}, \mathbf{p}\right)=f\left(\mathbf{p}^{\prime}, \mathbf{p}\right)$ for all $j$, then (3) would reduce to

$$
\frac{d \sigma}{d \Omega}=A^{2}\left|f\left(\mathbf{p}^{\prime}, \mathbf{p}\right)\right|^{2}
$$

Evidently, in that case, the coherent scattering cross-section would be enhanced by a factor of $A^{2}$ compared to that of a single constituent. In the realistic case of a nucleus with $Z$ protons and $N$ neutrons, and assuming zero nuclear spin, the corresponding differential cross-section reads [9]

$$
\frac{d \sigma}{d \Omega}=\frac{G_{F}^{2}}{4(2 \pi)^{2}} E^{2}(1+\cos \theta)\left[\left(1-4 s_{W}^{2}\right) Z-N\right]^{2},
$$




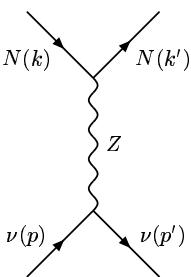

(a)

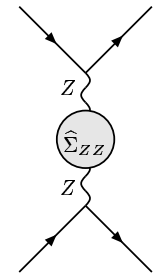

(b)

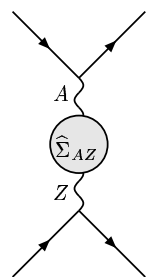

(c)

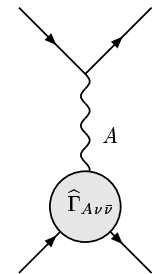

(d)
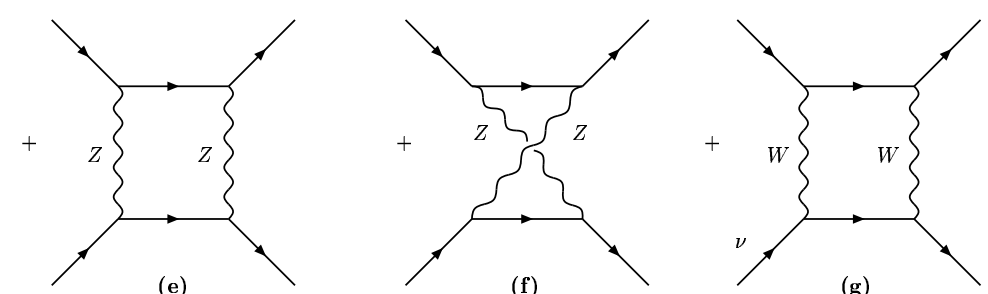

(g)

Figure 1: The various diagrams contributing to the one-loop amplitude. One-loop vertex corrections with an incoming $Z$ are omitted, because they are suppressed by a factor $q^{2} / M_{Z}^{2}$ [3].

where $s_{W}$ is the sine of the weak mixing angle, $d \Omega=d \phi d(\cos \theta)$, and $\theta$ is the scattering angle. For elastic scattering, the scattering angle is related to the nuclear recoil, so that the kinetic energy distribution of the recoiling nucleus is written as [9]

$$
\frac{d \sigma}{d y}=\frac{G_{F}^{2}}{2 \pi} \frac{M(M+2 E)^{2}}{[M+2 E(1-y)]^{3}} E^{2}(1-y)\left[\left(1-4 s_{W}^{2}\right) Z-N\right]^{2},
$$

where $M$ is the mass of the nucleus, $y=T / T_{\max }, y \in[0,1]$, and $T_{\max }=2 E^{2} /(M+2 E)$. For $2 E \ll M$, $T_{\max } \simeq 2 E^{2} / M$, and, to an excellent approximation, (5) simplifies to the rather compact expression,

$$
\frac{d \sigma}{d y} \simeq \frac{G_{F}^{2}}{2 \pi} E^{2}(1-y)\left[\left(1-4 s_{W}^{2}\right) Z-N\right]^{2} .
$$

The one-loop interactions between a nucleon $N$ and a neutrino $v$ are shown in Fig.1; of course, when the nucleon is a neutron, both (c) and (d) vanish. It is important to realize that, in the kinematic limit considered (i.e. $\left|q^{2}\right| \ll M^{2}$ ), all one-loop contributions may be absorbed into shifts of the original parameters appearing in the Born amplitude (7), giving rise to a Born-improved amplitude. In particular, as has been explained in detail in [3], diagrams (a), (b), and (c), combine to form two renormalization-group-invariant quantities,

$$
\bar{R}_{Z}\left(q^{2}\right)=\frac{\alpha_{W}}{c_{W}^{2}}\left[q^{2}-M_{Z}^{2}+\Re e\left\{\widehat{\Sigma}_{Z Z}\left(q^{2}\right)\right\}\right]^{-1}, \bar{s}_{W}^{2}\left(q^{2}\right)=s_{W}^{2}\left(1-\frac{c_{W}}{s_{W}} \mathfrak{R} e\left\{\widehat{\Pi}_{A Z}\left(q^{2}\right)\right\}\right)
$$

where $\alpha_{W}=g_{W}^{2} / 4 \pi, \mathfrak{R} e\{\ldots\}$ denotes the real part, and $\widehat{\Sigma}_{A Z}\left(q^{2}\right)=q^{2} \widehat{\Pi}_{A Z}\left(q^{2}\right) . \quad \bar{R}_{Z}\left(q^{2}\right)$ can be directly related to the effective electroweak (running) coupling, and eventually be interpreted as a shift to $G_{F}^{2}$. Similarly, $\bar{s}_{W}^{2}\left(q^{2}\right)$ defines the effective (running) weak mixing angle. It turns out that the UV-finite contribution from the NCR, contained in diagram (d), may be also absorbed into a 
shift of $s_{W}^{2}$. In fact, a detailed analysis based on the methodology developed in [10], reveals that, in the kinematic range of interest, the numerical impact of $\bar{R}_{Z}\left(q^{2}\right)$ and $\bar{s}_{W}^{2}\left(q^{2}\right)$ is negligible, i.e. these quantities do not run appreciably. Instead, the contribution from the NCR amounts to a correction of few percents to $s_{W}^{2}$, given by an expression of the form $s_{W}^{2} \longrightarrow s_{W}^{2}\left(1-\frac{2}{3} M_{W}^{2}\left\langle r_{v_{i}}^{2}\right\rangle\right)$. Finally, the contributions of the boxes are to be included. One can show that the sum of (e) and (f) vanishes in the relevant kinematic limit, whereas graph $(\mathrm{g})$ gives a contribution proportional to $g_{W}^{4} / M_{W}^{2}$, whose impact is currently under investigation. Finally we would like to point out that if one were to consider the differences in the cross-sections between two different neutrino species scattering coherently off the same nucleus, as proposed by Sehgal two decades ago [11], one would eliminate all unwanted contributions, such as boxes, thus measuring the difference between the two corresponding charge radii. Such a difference would also contribute to a difference for the neutrino index of refraction in nuclear matter [12].

\section{Acknowledgments}

This work was supported by the MCyT grant FPA2002-00612 and by the European Program MRTN-CT-2004-503369.

\section{References}

[1] J. L. Lucio, A. Rosado and A. Zepeda, Phys. Rev. D 29, 1539 (1984); N. M. Monyonko and J. H. Reid, Prog. Theor. Phys. 73, 734 (1985); A. Grau and J. A. Grifols, Phys. Lett. B 166, 233 (1986); P. Vogel and J. Engel, Phys. Rev. D 39, 3378 (1989); M. J. Musolf and B. R. Holstein, Phys. Rev. D 43, 2956 (1991); G. Degrassi, A. Sirlin and W. J. Marciano, Phys. Rev. D 39, 287 (1989).

[2] J. Bernabeu, L. G. Cabral-Rosetti, J. Papavassiliou and J. Vidal, Phys. Rev. D 62, 113012 (2000).

[3] J. Bernabeu, J. Papavassiliou and J. Vidal, Phys. Rev. Lett. 89, 101802 (2002) [Erratum-ibid. 89, 229902 (2002)]; Nucl. Phys. B 680, 450 (2004).

[4] J. Bernabeu, J. Papavassiliou and D. Binosi, Nucl. Phys. B 716, 352 (2005).

[5] J. M. Cornwall, Phys. Rev. D 26, 1453 (1982); J. M. Cornwall and J. Papavassiliou, Phys. Rev. D 40, 3474 (1989); J. Papavassiliou, Phys. Rev. D 41, 3179 (1990); G. Degrassi and A. Sirlin, Phys. Rev. D 46, 3104 (1992); M. Passera and K. Sasaki, Phys. Rev. D 54, 5763 (1996); A. Pilaftsis, Nucl. Phys. B 487, 467 (1997); D. Binosi and J. Papavassiliou, Phys. Rev. D 66, 111901 (2002).

[6] See, for example, S. Eidelman et al. [Particle Data Group], Phys. Lett. B 592 (2004), page 441.

[7] I. Giomataris et al., arXiv:hep-ex/0502033; Y. Giomataris and J. D. Vergados, arXiv:hep-ex/0503029.

[8] J. Barranco, O. G. Miranda and T. I. Rashba, arXiv:hep-ph/0508299.

[9] J. Bernabeu, Lett. Nuovo Cimento 10, 329 (1974); Astron. Astrophys. 47, 375 (1976);

D. Z. Freedman, Phys. Rev. D 9, 1389 (1974); D. Z. Freedman, D. N. Schramm and D. L. Tubbs, Ann. Rev. Nucl. Part. Sci. 27, 167 (1977); A. Drukier and L. Stodolsky, Phys. Rev. D 30, 2295 (1984).

[10] K. Hagiwara, S. Matsumoto, D. Haidt and C. S. Kim, Z. Phys. C 64, 559 (1994).

[11] L. M. Sehgal, Phys. Lett. B 162, 370 (1985).

[12] F. J. Botella, C. S. Lim and W. J. Marciano, Phys. Rev. D 35, 896 (1987); E. K. Akhmedov, C. Lunardini and A. Y. Smirnov, Nucl. Phys. B 643, 339 (2002) 